Received: 2 October 2017

Accepted: 4 January 2018

Published online: 18 January 2018

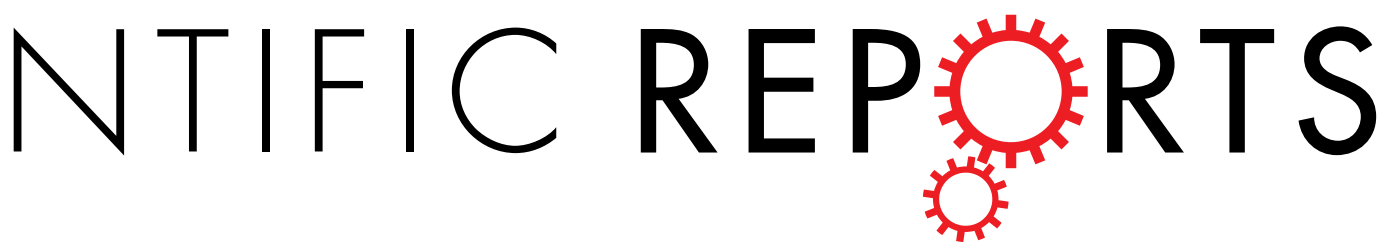

\title{
Intraocular inflammatory cytokines in patients with neovascular age- related macular degeneration before and after initiation of intravitreal injection of anti-VEGF inhibitor
}

Tomohito Sato ${ }^{1}$, Masaru Takeuchi $\mathbb{1}^{1}$, Yoko Karasawa ${ }^{1}$, Toshio Enoki ${ }^{3} \&$ Masataka Ito $^{2}$

Age-related macular degeneration (AMD) is a cause of blindness in people older than 50 years. Accumulating evidence indicates the involvement of systemic and local inflammation in the pathogenesis and progression of AMD. Aflibercept is an anti-vascular endothelial growth factor (VEGF) inhibitor, and intravitreal injection of aflibercept (IVA) is the approved treatments of neovascular AMD (nAMD), but the effect on inflammatory response remains unclear. The aim of our study was to investigate the profiles of inflammatory cytokines in the aqueous humor of $n A M D$ patients before and after initiation of IVA. In nAMD patients, IP-10 level was significantly higher and IL-6 level was significantly lower compared with those of cataract patients as controls. Logistic regression analysis identified IP-10 as a positive factor and IL- 6 a negative factor associated with the pathogenesis of nAMD. In addition, IP-10 level correlated positively with the mean thickness of macula in the central 1-mm diameter circle. After initiation of IVA, IP-10 level was further elevated, and correlated negatively with VEGF level. These data suggest that IP-10 plays a critical role as an antiangiogenic factor and at the same time an inflammatory factor in the pathogenesis and pathophysiology of nAMD eyes at onset and after IVA initiation.

Age-related macular degeneration (AMD) is a severe ocular disease affecting mainly elderly people, and is a major cause of blindness in people older than 50 years in developed countries ${ }^{1}$. Although the pathophysiology of AMD is not well understood, genetic variation, living environment and lifestyle have certain influence on the pathogenesis and progression of $\mathrm{AMD}^{2}$. AMD is broadly classified into two types according to the pathological mechanism; dry type and neovascular type ${ }^{3}$.

In neovascular AMD (nAMD), choroidal neovascularization (CNV) develops in the choroid, leading to suband intra-retinal macular edema, hemorrhage, and fibrosis responsible for visual decay ${ }^{4}$. Accumulating evidence indicates that systemic and ocular inflammation participates in the pathophysiology of $\mathrm{nAMD}^{5,6}$. Therefore, nAMD has been recognized not only as an exudative vascular event but also a chronic inflammatory disease in the retina and choroid ${ }^{1,7}$.

Vascular endothelial growth factor (VEGF) is a crucial mediator of angiogenesis and vascular permeability, and contributes to the development of choroidal neovascularization ${ }^{5}$. In mammals, the VEGF family consists of VEGF-A, VEGF-B, VEGF-C, VEGF-D and placenta growth factor (PlGF) ${ }^{8}$. VEGF-A and PlGF are signal proteins that promote angiogenesis and vascular leakage in the retina ${ }^{9}$. VEGF initiates biological effects by binding three transmembrane receptors; VEGF receptor (VEGFR)-1, VEGFR-2 and VEGFR-3 ${ }^{10}$. VEGF receptors are

${ }^{1}$ Department of Ophthalmology, National Defense Medical College, Tokorozawa, Saitama, Japan. ${ }^{2}$ Department of Developmental Anatomy and Regenerative Biology, National Defense Medical College, Tokorozawa, Saitama, Japan. ${ }^{3}$ Enoki Eye Clinic, Sayama, Saitama, Japan. Correspondence and requests for materials should be addressed to M.T. (email: masatake@ndmc.ac.jp) 


\begin{tabular}{|l|l|l|l|}
\hline Characteristic & nAMD patients $(\mathbf{n}=\mathbf{2 1})$ & Control $(\mathbf{n}=17)$ & P value \\
\hline Age $($ year $)$ & $69.0 \pm 10.2^{*}(48 \sim 8)$ & $73.7 \pm 9.43(56 \sim 90)$ & 0.12 \\
\hline Gender (M/F) & $15 / 6$ & $6 / 11$ & 0.06 \\
\hline phenotypes & \multicolumn{3}{|l|}{} \\
\hline CNV Type I & $4\left(19^{\dagger}\right)$ & & \\
\hline CNV Type II & $9(43)$ & & \\
\hline PCV & $8(38)$ & & \\
\hline RAP & $0(0)$ & & \\
\hline
\end{tabular}

Table 1. Clinical characteristics of nAMD patients and controls. nAMD: Neovascular age-related macular degeneration. CNV: Choroidal neovascularization. PCV: Polypoidal choroidal vasculopathy. RAP: Retinal angiomatous proliferation. *Mean \pm standard deviation (range). ${ }^{\dagger}$ Percentage.

expressed on various types of cells including vascular endothelial cells, pericytes, monocytes and macrophages ${ }^{11}$. VEGF-A recruits monocytes and macrophages by binding VEGFR-1 in the process of inflammatory neovascularization $^{12}$. Since ocular fluid levels of VEGF increase markedly in nAMD patients, intravitreal injection of anti-VEGF agents has been the primary treatment for $\mathrm{nAMD}{ }^{4,13}$. Aflibercept is an anti-VEGF agent and a fusion protein containing human VEGFR-1 and VEGFR-2 that bind VEGF-A, VEGF-B and PlGF. Intravitreal injection of aflibercept (IVA) is currently one of the promising treatments for nAMD ${ }^{14}$.

Aqueous humor and vitreous fluid reflect intraocular immune conditions of nAMD more directly than serum. Sampling of aqueous humor is easier and safer than sampling vitreous fluid. Furthermore, aqueous humor levels of cytokines have been proposed to reflect the levels in the vitreous cavity, and correlation between cytokines levels in aqueous humor and vitreous fluid has been reported ${ }^{15,16}$. Several papers that compared cytokine levels between $n A M D$ patients and controls by nonparametric statistical tests indicated an association of aqueous humor cytokines with the pathogenesis of nAMD. Cha et al. ${ }^{17}$ reported that altered expression of insulin-like growth factor (IGF)-related molecules including IGF binding protein (IGFBP)-2 and IGF-1 may be involved in the pathogenesis of nAMD and suggested that these molecules are potential biomarkers for nAMD. Agawa et al. ${ }^{5}$ proposed that VEGF, angiogenin, interferon gamma-inducible protein (IP)-10, monocyte chemotactic protein (MCP)-1, macrophage inflammatory protein (MIP)-1 $\beta$, and monokine induced by interferon $\gamma$ (Mig) may be related to nAMD. Sakurada et al. ${ }^{18}$ also showed that IP-10 was associated with the pathogenesis of nAMD and polypoidal choroidal vasculopathy.

The purpose of this study was to investigate the association of aqueous humor cytokines with the pathogenesis of nAMD, and to evaluate the association of aqueous humor cytokine levels with clinical findings before and after initiation of IVA in eyes with nAMD.

\section{Methods}

Subjects. This prospective observational study enrolled 21 eyes of 21 nAMD patients (nAMD group) and 22 eyes of 17 cataract patients (control group). The study was performed at National Defense Medical College Hospital and Enoki Eye Clinic in Japan between August 1, 2013 and July 30, 2015. This study protocol was approved by the Ethics Committee of National Defense Medical College, and the procedures conformed to the tenets of the Declaration of Helsinki. Informed consent was obtained from all patients before enrolling in this study. The clinical characteristics of nAMD group and control group are summarized in Table 1 . There were no significant differences in age and gender ratio between $\mathrm{nAMD}$ group and control group.

Inclusion criteria for nAMD patients in this study were: (1) patients older than 50 years; (2) previously untreated CNV; (3) OCT showing intraretinal edema, subretinal fluid, or pigment epithelial detachment; and (4) absence of concurrent ocular diseases in the study eye, which had compromised or could have compromised vision and ocular condition. Exclusion criteria were: (1) clinical features suggesting that CNV was secondary to other causes such as pathologic myopia, trauma and hereditary diseases; (2) myopia greater than -6 diopter; (3) a history of treatment for $\mathrm{nAMD}$, including intravitreal drug injection, photodynamic therapy, systemic or topical steroids; (4) previous intraocular surgery except cataract surgery, which had been performed more than 6 months before enrolment in the study.

Diagnostics and Treatments. Diagnosis of nAMD was based on a full ophthalmological examination including best corrected visual acuity (BCVA) test using a decimal chart, slit-lamp biomicroscopy, dilated fundus examination, intraocular pressure (IOP) measurement, color fundus photography, fundus fluorescein angiography, fundus indocyanine green angiography and spectral-domain (SD) OCT (Cirrus HD-OCT; Carl Zeiss Meditec, Dublin, CA, USA). Neovascular AMD was classified according to the classification and diagnostic criteria of $\mathrm{AMD}^{3,19}$. Four eyes (19.0\%) in the nAMD group was classified as type I neovascularization, 9 eyes $(42.9 \%)$ as type II neovascularization, 8 eyes $(38.0 \%)$ as polypoidal choroidal vasculopathy, and 0 eye $(0 \%)$ as retinal angiomatous proliferation (Table 1). BCVA was converted to logMAR units for statistical analysis. For measurement of retinal thickness, a traditional Early Treatment Diabetic Retinopathy Study (ETDRS) grid containing three concentric rings 1,3 , and $6 \mathrm{~mm}$ in diameter was employed ${ }^{20}$. The mean retinal thicknesses of the macula in the central 1, 3 and $6 \mathrm{~mm}$ on the ETDRS grid were defined as CMT $\leq 1 \mathrm{~mm}, \mathrm{CMT} \leq 3 \mathrm{~mm}$, and CMT $\leq 6 \mathrm{~mm}$, respectively ${ }^{21}$. During the study period of 8 weeks, follow-up examinations were performed before the first IVA and after the third IVA. 

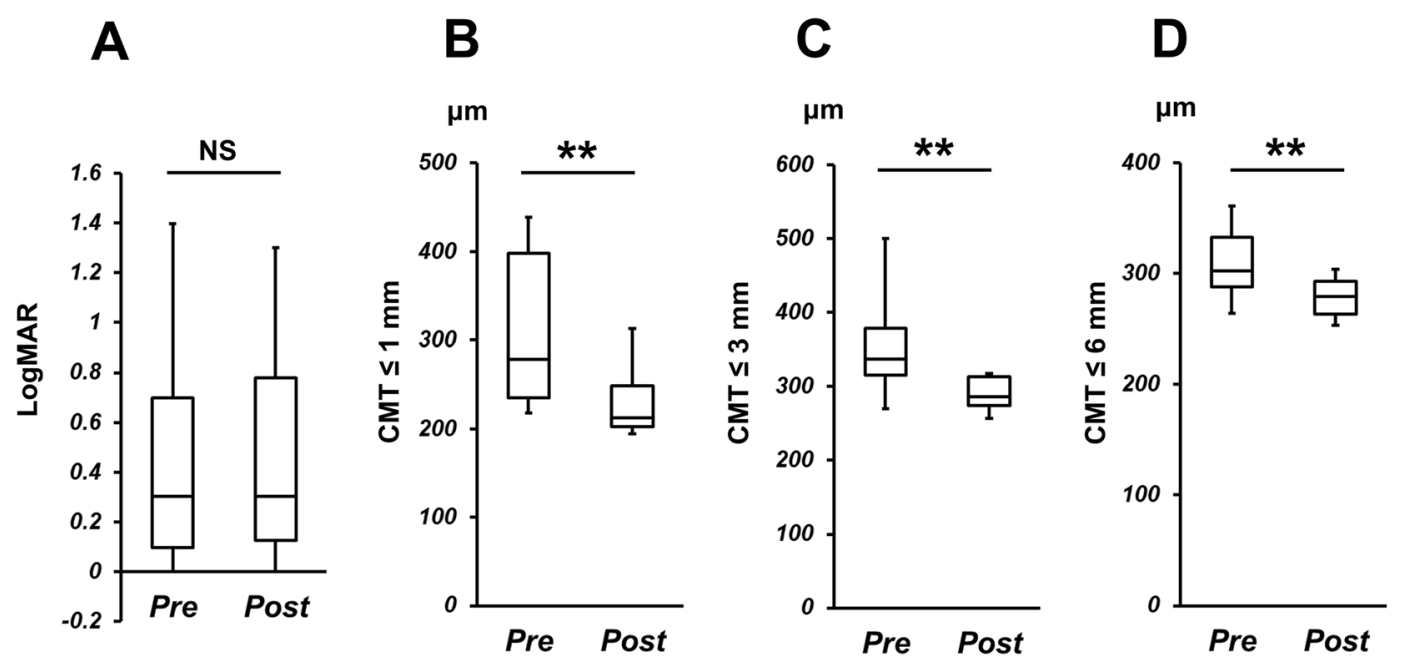

Figure 1. LogMAR and central macular thickness (CMT). (A) LogMAR, (B) CMT $\leq 1 \mathrm{~mm},(\mathbf{C}) \mathrm{CMT} \leq 3 \mathrm{~mm}$, and (D) CMT $\leq 6 \mathrm{~mm}$ in control group (Cont), and in nAMD group before (nAMD pre) and after initiation of IVA therapy (nAMD post). NS: Not significant, $* * P<0.01$.

Intravitreal Injection of Aflibercept and Aqueous Humor Sample Collection. All nAMD patients were given intravitreal injection of $2 \mathrm{mg}$ aflibercept every 4 weeks for 2 months (a total of three IVA) according to the protocol reported previously. $y^{5}$ Before the first IVA (pre-IVA) and before the third IVA (post-IVA) in nAMD group, approximately $0.1 \mathrm{~mL}$ of undiluted aqueous humor was collected by performing an anterior chamber limbal paracentesis. Control samples of undiluted aqueous humor were obtained at the beginning of cataract surgery. The aqueous humor samples were transferred into sterile tubes and stored at $-80^{\circ} \mathrm{C}$ until processing. No complications associated with aqueous humor sampling occurred.

Cytokine Measurements. Twenty-seven cytokines in aqueous humor samples were measured by a Bio-Plex multiplex assay (Bio-Plex Human Cytokine 27-plex panel; Bio-Rad, Hercules, CA, USA) and a multiplex bead analysis system (Bio-Plex Suspension Array System; Bio-Rad) according to manufacturers' instructions with standards and samples in duplicate. The lower limits of detection for the 27 cytokines were as follows [mean (range)]: 4.18 (1.68-7.37) pg/ml for platelet derived growth factor BB (PDGF-BB); $1.25(0.53-1.99) \mathrm{pg} / \mathrm{ml}$ for IL-1 $\beta ; 6.03$ (5.73-6.47) pg/ml for IL-1 receptor antagonist (IL-1ra); $1.07(0.93-1.21) \mathrm{pg} / \mathrm{ml}$ for IL-2; $0.48(0.21-$ $1.15) \mathrm{pg} / \mathrm{ml}$ for IL-4; 1.41 (1.15-1.62) pg/ml for IL-5; $1.83(1.38-2.28) \mathrm{pg} / \mathrm{ml}$ for IL-6; $1.62(0.98-2.18) \mathrm{pg} / \mathrm{ml}$ for IL-7; 2.25 (1.91-2.58) $\mathrm{pg} / \mathrm{ml}$ for IL-8; $1.60(0.57-2.32) \mathrm{pg} / \mathrm{ml}$ for IL-9; 2.14 (2.14-2.15) pg/ml for IL-10; 2.37 (2.27-2.44) $\mathrm{pg} / \mathrm{ml}$ for IL-12; $1.25(0.45-2.03) \mathrm{pg} / \mathrm{ml}$ for IL-13; $1.43(1.37-1.49) \mathrm{pg} / \mathrm{ml}$ for IL-15; $3.56(1.62-5.93)$ $\mathrm{pg} / \mathrm{ml}$ for IL-17A; $3.05(1.26-6.8) \mathrm{pg} / \mathrm{ml}$ for eotaxin; $2.70(0.78-3.85) \mathrm{pg} / \mathrm{ml}$ for basic fibroblast growth factor (bFGF); $2.16(1.90-2.41) \mathrm{pg} / \mathrm{ml}$ for granulocyte colony-stimulating factor (G-CSF); $2.94 \mathrm{pg} / \mathrm{ml}(0.63-4.01 \mathrm{pg} /$ $\mathrm{ml}$ ) for Granulocyte-macrophage colony-stimulating factor (GM-CSF); $9.80(5.06-21.21) \mathrm{pg} / \mathrm{ml}$ for IFN- $\gamma ; 8.34$ (7.36-9.18) $\mathrm{pg} / \mathrm{ml}$ for IP-10; 1.49 (1.29-1.67) pg/ml for MCP-1; $0.83(0.28-1.05) \mathrm{pg} / \mathrm{ml}$ for MIP- $1 \alpha ; 2.18(0.53-$ $3.77) \mathrm{pg} / \mathrm{ml}$ for MIP-1 $\beta ; 2.09(1.03-4.11) \mathrm{pg} / \mathrm{ml}$ for regulated on activation, normal T-cell expressed and secreted (RANTES); 4.76 (4.05-5.64) pg/ml for tumor necrosis factor $\alpha$ (TNFo); and 2.04 (1.63-2.83) pg/ml for VEGF. Levels of aqueous humor cytokines below detectable levels were treated as 0 for statistical analysis ${ }^{5}$.

Statistical Analysis. Statistical analyses were performed using the statistic add-in software for Excel (SSRI Co., Ltd., Tokyo, Japan). Data are expressed as mean \pm standard deviation (SD). Yates' chi-squared test (for $\mathrm{n}<10$ ) or Fisher's exact test (for $\mathrm{n}<4$ ) was used to compare categorical variables. Mann-Whitney $U$ test was used to compare aqueous humor levels of cytokines between nAMD group and control group. Wilcoxon signed rank test was used to compare aqueous humor levels of cytokines before the first IVA (pre-IVA) and before the third IVA (post-IVA) in nAMD group. Spearman's correlation analysis was used to evaluate the relationship between numerical data. To examine the association of elevated or decreased cytokines with the pathogenesis of nAMD, logistic regression analysis was performed using nAMD or not as response variable and cytokines with high detectable rates of over $80 \%$ in pre-IVA of nAMD and control groups and significant differences between them as explanatory valuables. A $P$ level less than 0.05 was considered to be statistically significant.

Data Availability. All data presented in the present study are available from the corresponding author on reasonable request.

\section{Results}

The profiles of logMAR and mean macular thickness in nAMD group before and after initiation of IVA therapy are shown in Fig. 1. LogMAR was $0.51 \pm 0.60$ before IVA and $0.48 \pm 0.67$ after IVA, and there was no significant difference between them $(P=0.33)$. On the other hand, CMT $\leq 1 \mathrm{~mm}, \mathrm{CMT} \leq 3 \mathrm{~mm}$, and CMT $\leq 6 \mathrm{~mm}$ were $345 \pm 201 \mu \mathrm{m}, 384 \pm 150 \mu \mathrm{m}$, and $321 \pm 63.7 \mu \mathrm{m}$ before IVA, and were $256 \pm 129 \mu \mathrm{m}, 307 \pm 82.3 \mu \mathrm{m}$, and 


\begin{tabular}{|c|c|c|c|c|c|c|c|c|c|}
\hline & \multicolumn{4}{|c|}{ nAMD $(n=21)$} & \multirow{2}{*}{\multicolumn{2}{|c|}{ Controls $(n=17)$}} & \multirow[b]{3}{*}{ Pre IVA } & \multirow{2}{*}{\multicolumn{2}{|c|}{$P$ value }} \\
\hline & \multicolumn{2}{|c|}{ pre-IVA } & \multicolumn{2}{|l|}{ post-IVA } & & & & & \\
\hline & Detectable & Level & Detectable & Level & Detectable & Level & & Post IVA & Pre IVA \\
\hline & samples* & mean \pm SD & samples & mean \pm SD & samples & mean \pm SD & vs Control & vs Control & vs Post IVA \\
\hline IL-1r $\alpha$ & $0(0)^{*}$ & 0 & $1(4.76)$ & $0.70 \pm 3.23^{\dagger}$ & $1(5.88)$ & $0.77 \pm 3.19$ & 0.381 & 0.483 & 0.164 \\
\hline IL-6 & $16(76.2)$ & $6.51 \pm 5.24$ & $18(85.7)$ & $12.6 \pm 13.7$ & $15(88.2)$ & $78.2 \pm 100$ & 0.0029 & 0.011 & 0.0098 \\
\hline IL-7 & $21(100)$ & $10.9 \pm 3.95$ & $21(100)$ & $12.4 \pm 6.67$ & $17(100)$ & $13.5 \pm 12.8$ & 0.465 & 0.397 & 0.219 \\
\hline IL-8 & $12(57.1)$ & $6.00 \pm 6.69$ & $14(66.7)$ & $10.3 \pm 11.7$ & $11(64.7)$ & $6.43 \pm 6.84$ & 0.448 & 0.177 & 0.087 \\
\hline IL-9 & $1(4.76)$ & $0.087 \pm 0.40$ & $1(4.76)$ & $0.18 \pm 0.81$ & $1(5.88)$ & $0.10 \pm 0.41$ & 0.483 & 0.483 & 0.328 \\
\hline IL-12 & $20(95.2)$ & $12.1 \pm 5.79$ & $0(0)$ & 0 & $12(70.6)$ & $10.4 \pm 9.11$ & 0.268 & $3.44 \times 10^{-4}$ & $4.45 \times 10^{-5}$ \\
\hline IL-13 & $12(57.1)$ & $1.97 \pm 2.22$ & $11(52.4)$ & $1.92 \pm 2.59$ & $11(64.7)$ & $2.25 \pm 2.65$ & 0.448 & 0.316 & 0.398 \\
\hline Eotaxin & $11(52.4)$ & $3.76 \pm 4.17$ & $11(52.4)$ & $3.66 \pm 4.04$ & $1(5.88)$ & $1.22 \pm 2.39$ & 0.035 & 0.043 & 1 \\
\hline$\beta F G F$ & $0(0)$ & 0 & $1(4.76)$ & $0.76 \pm 3.49$ & $1(4.55)$ & $0.71 \pm 2.92$ & 0.381 & 0.483 & 0.164 \\
\hline G-CSF & $1(4.76)$ & $0.28 \pm 1.27$ & $0(0)$ & 0 & $0(0)$ & 0 & 0.402 & - & 0.164 \\
\hline IP-10 & $21(100)$ & $755 \pm 645$ & $21(100)$ & $1844 \pm 2719$ & $17(100)$ & $273 \pm 260$ & $3.00 \times 10^{-4}$ & $6.85 \times 10^{-5}$ & 0.0026 \\
\hline MCP-1 & $21(100)$ & $229 \pm 155$ & $21(100)$ & $182 \pm 143$ & $17(100)$ & $204 \pm 112$ & 0.381 & 0.184 & 0.085 \\
\hline MIP- $1 \alpha$ & $4(19.0)$ & $0.41 \pm 0.91$ & $5(23.8)$ & $0.64 \pm 1.29$ & $4(23.5)$ & $0.96 \pm 2.35$ & 0.4 & 0.494 & 0.122 \\
\hline MIP-1 $\beta$ & $21(100)$ & $37.2 \pm 15.6$ & $21(100)$ & $38.3 \pm 19.6$ & $17(100)$ & $37.2 \pm 31.1$ & 0.112 & 0.197 & 0.372 \\
\hline VEGF & $21(100)$ & $228 \pm 176$ & $2(9.52)$ & $1.08 \pm 3.46$ & $17(100)$ & $132 \pm 54.2$ & 0.029 & $3.14 \times 10^{-6}$ & $2.98 \times 10^{-5}$ \\
\hline
\end{tabular}

Table 2. Aqueous humor levels of cytokines in controls and in nAMD patients before or after initiation of IVA therapy. nAMD: Neovascular age-related macular degeneration. IVA: Intravitreal injection of aflibercept. ${ }^{*}$ Number (\%) of samples with detectable cytokine. ${ }^{\dagger}$ Mean \pm standard deviation (pg/ml).

\begin{tabular}{|l|l|l|l|}
\hline & OR & $\mathbf{9 5} \%$ CI & $P$ value \\
\hline IL-6 & 0.920 & $(0.848-0.997)$ & 0.043 \\
\hline IP-10 & 1.004 & $(1.0007-1.007)$ & 0.018 \\
\hline VEGF & 1.009 & $(1.0003-1.019)$ & 0.043 \\
\hline
\end{tabular}

Table 3. Logistic regression analysis of the association of nAMD with cytokines in aqueous humor. nAMD: Neovascular age-related macular degeneration. $\mathrm{OR}=$ Odds ratio. $\mathrm{CI}=$ Confidence interval.

$284 \pm 33.0 \mu \mathrm{m}$ after IVA, respectively. There were significant differences in CMT $\leq 1 \mathrm{~mm}, \mathrm{CMT} \leq 3 \mathrm{~mm}$, and $\mathrm{CMT} \leq 6 \mathrm{~mm}$ before and after IVA $\left(P=2.11 \times 10^{-4}, 2.98 \times 10^{-5}\right.$, and $3.48 \times 10^{-5}$, respectively $)$.

Aqueous Humor Cytokine Levels in Control Group and in nAMD Group Before and After IVATherapy. The profiles of aqueous humor levels of cytokines detected in nAMD group before and after the initiation of IVA therapy, and in control group are shown in Table 2. In nAMD group before IVA, IL-6 level was significantly lower $(P=0.0029)$, but Eotaxin, IP-10 and VEGF levels were significantly higher $(P=0.035, P<0.001$ and $P=0.029$, respectively) compared to control group. Even after initiation of IVA, IL-6 level remained significantly lower $(P=0.011)$, and Eotaxin and IP-10 levels were significantly higher $(P=0.043$ and $P<0.001$, respectively) compared to control group. When comparing pre-IVA and post-IVA levels in nAMD group, IL-6 and IP-10 levels were elevated $(P=0.0098$ and $P=0.0026$, respectively), while IL-12 and VEGF levels were reduced remarkably (both $P<0.001$ ). In logistic regression analysis using nAMD or not as response variable and aqueous humor levels of IL-6, IP-10 and VEGF in control and pre-IVA of nAMD groups as explanatory valuables, it was also demonstrated that pre-IVA IP-10 and VEGF levels were significant higher $(P=0.018$ and $P=0.043$, respectively) and IL-6 level was significant lower $(P=0.043)$ in nAMD group than those in control group (Table 3 ).

Correlation of logMAR and Central Macular Thickness with Aqueous Humor IL-6, IP-10 and VEGF Levels in nAMD Patients. Based on the results of Table 2, we next examined the associations between clinical findings and aqueous humor IL-6, IP-10, and VEGF levels before and after initiation of IVA therapy in nAMD group. Figure 2 shows correlation between logMAR and aqueous humor level of IL-6, IP-10, or VEGF before and after initiation of IVA. Before IVA therapy, there was no significant correlation between logMAR and IL-6, IP-10, or VEGF level (Fig. 2A-C). However, a positive correlation $(P=0.034)$ between IL-6 level and $\operatorname{logMAR}$ was observed after initiation of IVA therapy (Fig. 2D), although there was no significant correlation between logMAR and IP-10 or VEGF (Fig. 2E and F).

Correlation of CMT with aqueous humor levels of IL-6, IP-10, or VEGF in nAMD group before IVA therapy is shown in Fig. 3 and the correlation after IVA therapy is presented in Fig. 4. A positive correlation was observed between pre-IVA level of IP-10 and CMT $\leq 1 \mathrm{~mm}(P=0.014)$ (Fig. 3B), although no significant correlation was found between pre-IVA IL- 6 or VEGF level and CMT $\leq 1 \mathrm{~mm}$ (Fig. 3A and C). There was no significant correlation between pre-IVA level of IL-6, IP-10 or VEGF and CMT $\leq 3 \mathrm{~mm}$ or CMT $\leq 6 \mathrm{~mm}$ (Fig. 3D-I). For the analysis of post-IVA levels, there was no significant correlation between IL-6, IP-10, or VEGF level and CMT $<1 \mathrm{~mm}$, $\mathrm{CMT} \leq 3 \mathrm{~mm}$, or $\mathrm{CMT} \leq 6 \mathrm{~mm}$ (Fig. $4 \mathrm{~A}-\mathrm{I})$. 

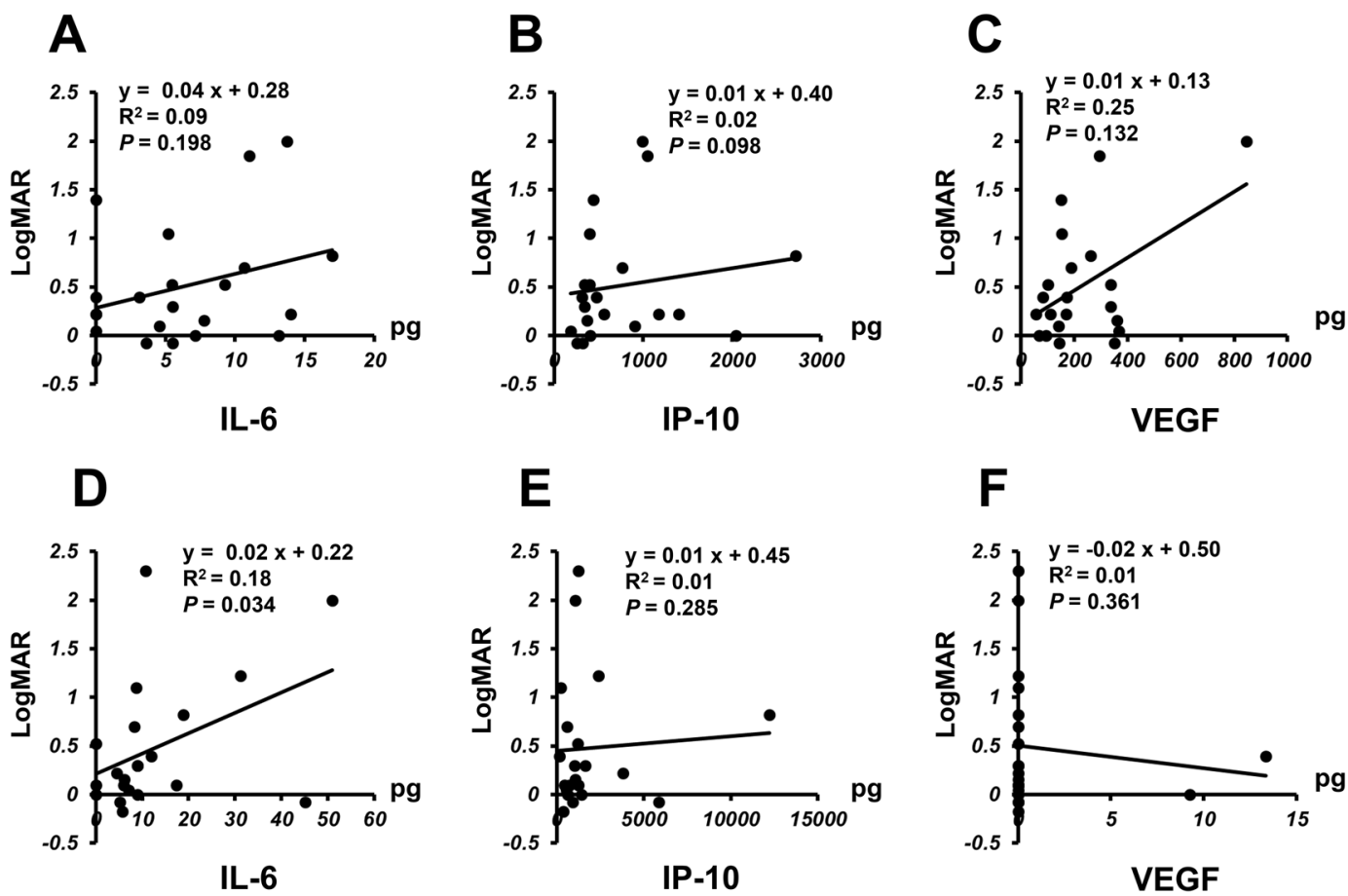

Figure 2. Relationship between logMAR and aqueous humor level of IL-6, IP-10 or VEGF in nAMD group before and after initiation of IVA therapy. Correlation between logMAR and aqueous humor level of IL-6, IP-10 or VEGF in nAMD group before (pre-IVA) (A-C) and after initiation of IVA therapy (post-nAMD) (D-F).

Correlation among Levels of IL-6, IP-10 and VEGF in nAMD Patients at Pre-IVA and Post-IVA. Figure 5 shows the correlation among pre-IVA and post-IVA levels of IL-6, IP-10, and VEGF in nAMD group. For the comparisons of pre-IVA levels, a positive correlation was observed between IL-6 level and IP-10 level $(P=0.029)$ (Fig. 5A), but no significant correlation was detected between VEGF level and IL-6 or IP-10 level (Fig. 5B and C). In post-IVA, there was a negative correlation between IP-10 level and VEGF level $(P=0.045)$, but no significant correlation between IL-6 level and IP-10 or VEGF level (Fig. 5D-F).

\section{Discussion}

The major findings of the present study were as follows. (1) High levels of VEGF and IP-10, and low level of IL-6 were the characteristic profile of inflammatory cytokines in aqueous humor of nAMD patients, (2) IVA reduced VEGF level and increased IL-6 level, but further increased IP-10 level in aqueous humor, with a negative correlation between IP-10 and VEGF levels. (3) Increased IL-6 level in aqueous humor of nAMD patients after initiation of IVA treatment negatively correlated with BCVA, and IP-10 level correlated with CMT $\leq 1 \mathrm{~mm}$ before but not after initiation of IVA.

AMD is a leading cause of irreversible blindness in people aged 50 years or older living in the developed world ${ }^{1}$. Genetic variation, living environment and lifestyle have been known to be risk factors in the pathogenesis and progression of $\mathrm{AMD}^{1}$. Previous studies on $\mathrm{nAMD}$ patients showed that complement components in plasma were activated, and the levels of proinflammatory cytokines in aqueous humor were significantly higher compared with those in cataract patients as controls $s^{5,6,22,23}$. Therefore, nAMD has been recognized not only an exudative vascular disease but also a chronic inflammatory disease in the retina and choroid ${ }^{1,7,24}$.

The aqueous humor reflects the immunological conditions of the eye more directly than serum, and has been used as the major intraocular sample because it can be collected repeatedly and more conveniently than vitreous fluid. Boyd et al. ${ }^{25}$ reported that aqueous humor VEGF level correlated significantly with that of vitreous fluid. Therefore, we selected aqueous humor cytokines as biomarkers that indicate the immunological features of eyes with nAMD.

In this study, BCVA did not improve significantly, but tended to recover by 2 sessions of IVA in nAMD patients, although CMT was reduced significantly. VIEW 2 study reported that IVA every 4 weeks improved visual acuity by approximately 7 ETDRS letters after 8 weeks, and the degree of BCVA improvement was almost equivalent to a decrease of $0.14 \log \mathrm{MAR}^{26}$. The numbers of subjects enormously affect statistical analysis, and VIEW 2 study is a much larger clinical study compared with the present study. In addition, the inclusion criteria of VIEW 2 study are different from our study. In VIEW 2 study, the inclusion criteria are as follows: (1) CNV comprised at least $50 \%$ of total lesion size; (2) BCVA before IVA was within a range of 25 to 73 ETDRS letters, almost equivalent to logMAR 0.301 to 0.164 . In our study, the CNV lesion size and BCVA were not restricted, and nAMD patients with a wider range of conditions are enrolled in this study as shown in Fig. 2. Therefore, two sessions of IVA might be not sufficient in treatment duration and/or treatment frequency to improve BCVA significantly. 

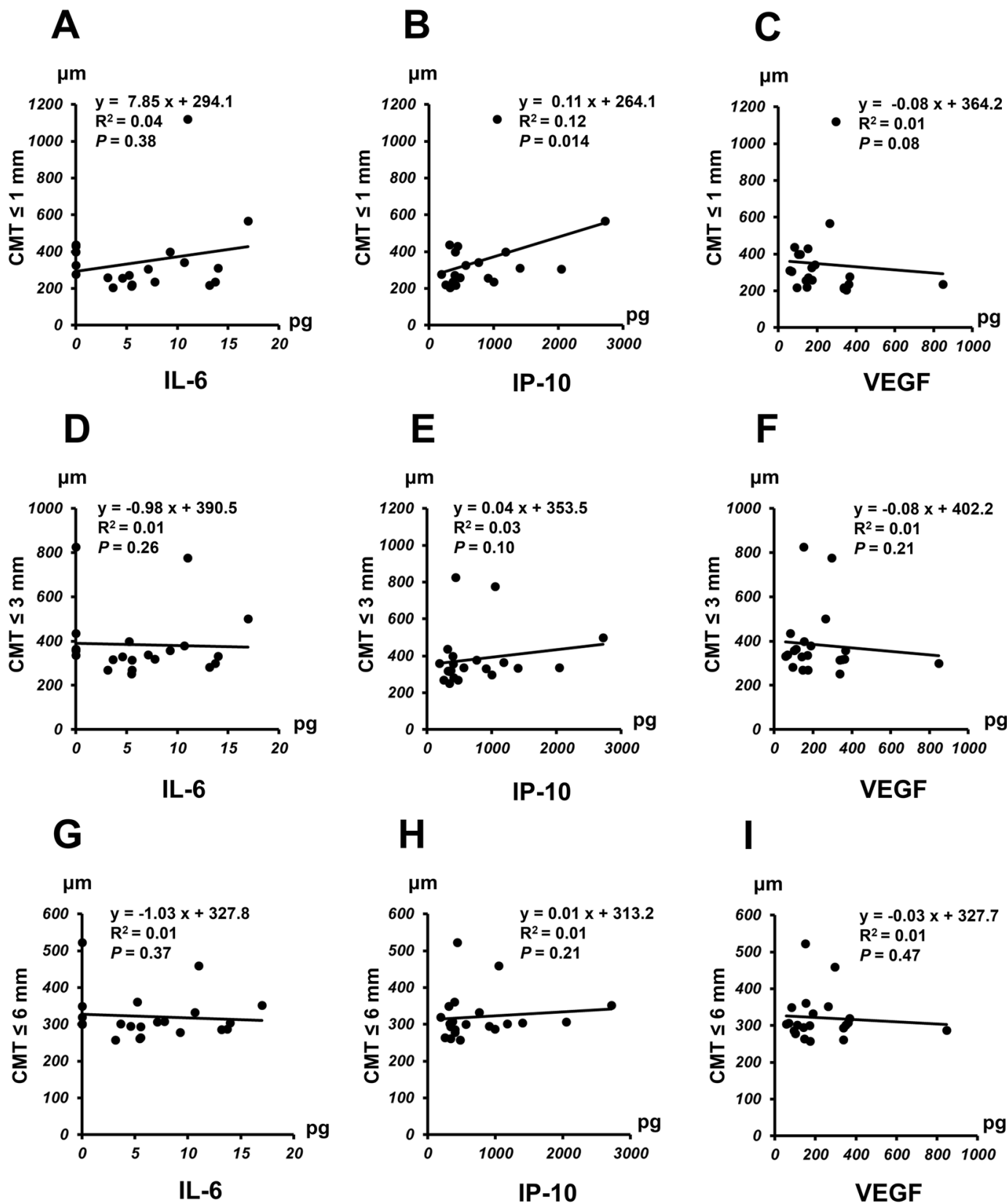

Figure 3. Relationship between central macular thickness (CMT) and aqueous humor level of IL-6, IP-10 or VEGF in nAMD group before initiation of IVA therapy. Correlation of CMT $\leq 1 \mathrm{~mm}(\mathbf{A}-\mathbf{C}), \mathrm{CMT} \leq 3 \mathrm{~mm}$ (D-F), or CMT $\leq 6(\mathbf{G}-\mathbf{I}) \mathrm{mm}$ with pre-IVA aqueous humor level of IL-6, IP-10 or VEGF in nAMD group.

Our study showed that IP-10 was elevated in the aqueous humor of nAMD patients, and IVA further increased the IP-10 level. IP-10 is secreted by leukocytes such as monocytes and macrophages, and by non-leukocytes including endothelial cells and smooth muscle cells ${ }^{27}$. Regarding response to neovascularization, IP-10 has antiangiogenic and antifibrotic actions ${ }^{28,29}$. Bodnar et al..$^{30}$ reported that IP-10 inhibited VEGF-induced endothelial cell motility and tube formation in vitro. In postmortem eyes with early AMD, IP-10 was strongly expressed in neovascular endothelial cells and connective tissue matrix associated with $\mathrm{CNV}^{31}$. Boulday et al. ${ }^{32}$ reported that VEGF induced over-expression of IP-10 in endothelial cells in vitro and in vivo. Findings of the present study and previous reports thus suggest that aflibercept may induce IP-10 production that exhibits antiangiogenic effects to inhibit the development of $\mathrm{CNV}$ at the onset of nAMD.

Higher level of IP-10 in aqueous humor of nAMD was associated with greater CMT before IVA therapy, although decrease of CMT following IVA abolished the association. Sakamoto et al. also measured IP-10 level in the aqueous humor of nAMD patients before first and third intravitreal injection of ranimizumab (IVR), and found that the aqueous humor level of IP-10 in nAMD patients was higher than that of controls although IP-10 was not increased after the treatment with $\mathrm{IVR}^{33}$. In addition, they indicated that IP-10 level in the aqueous humor was associated with initial CRT by multiple regression analysis, as well as our present results. 

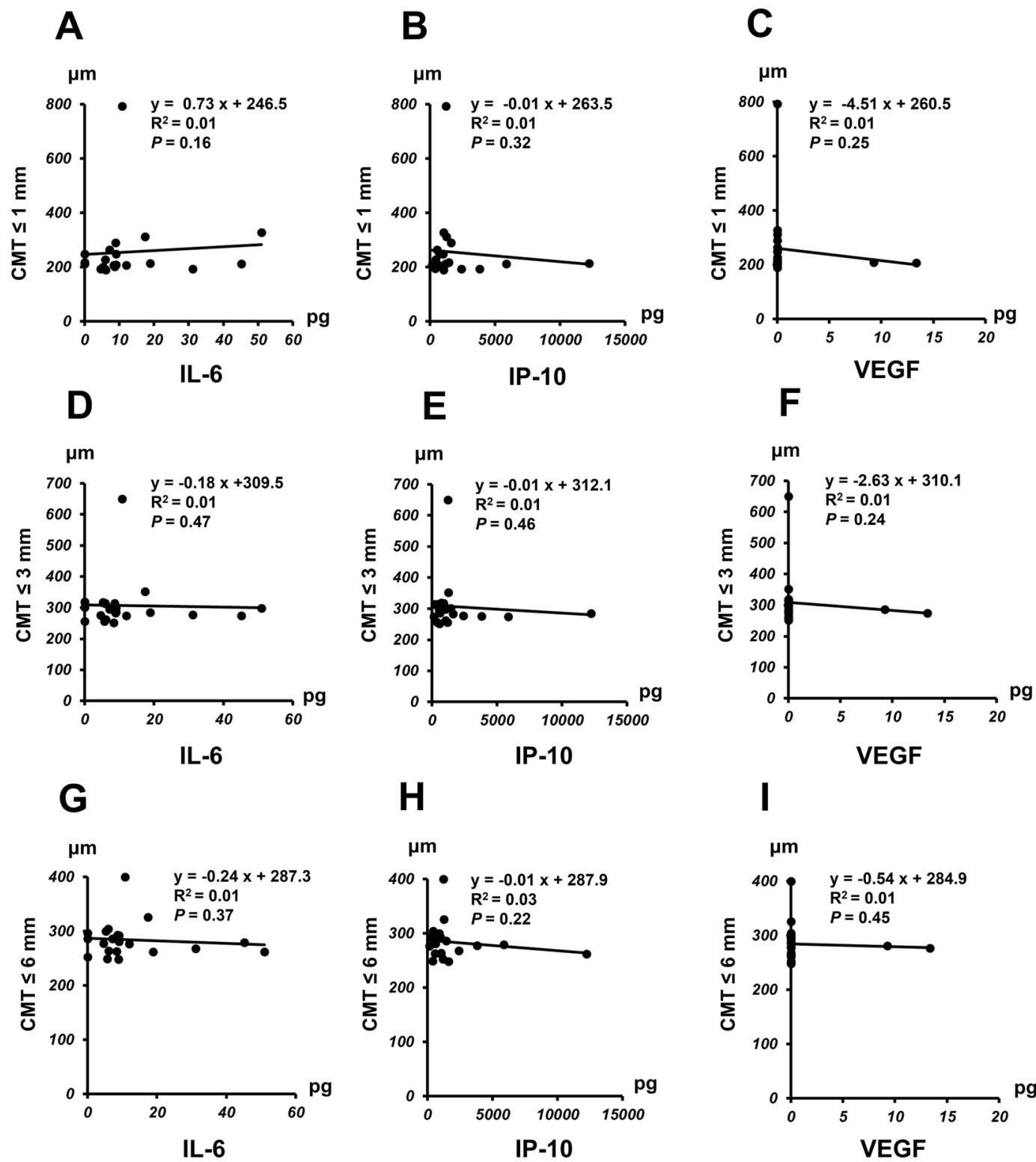

Figure 4. Relationship between central macular thickness (CMT) and aqueous humor level of IL-6, IP-10 or VEGF in nAMD group after initiation of IVA therapy. Correlation of CMT $\leq 1 \mathrm{~mm}(\mathbf{A}-\mathbf{C}), \mathrm{CMT} \leq 3 \mathrm{~mm}(\mathbf{D}-\mathbf{F})$, or CMT $\leq 6 \mathrm{~mm}(\mathbf{G}-\mathbf{I})$ with post-IVA aqueous humor level of IL-6, IP-10 or VEGF in nAMD group.

IP-10 is a specific chemotactic factor for Th1 cells, and activates Th1 cell-mediated immune response via binding the common CXC chemokine receptor (CXCR) $3^{27,34}$. Previous in vitro and in vivo studies reported that CCR3 and CXCR3 were involved in the development of nAMD, and CCR2 and CX3CR1 were associated with drusen formation in early $\mathrm{AMD}^{35-38}$. Examination of postmortem eyes with $\mathrm{nAMD}$ revealed overexpression of helper T-cell-related cytokines in the retina ${ }^{31,39}$. Singh et al. ${ }^{40}$ suggested the involvement of the adaptive immune system in AMD, and proposed AMD as a systemic disease. Therefore, it is possible that low-grade inflammation related to high level of IP-10 induces increase in central macular thickness in nAMD, independent of the development of CNV.

On the other hand, angiogenesis is arrested during the regenerative phase of wound healing ${ }^{30}$. After IVA treatment, CNV may also be attenuated, and the regenerative and remodeling process may be initiated in the CNV-damaged retina. IP-10 is produced by vascular endothelial cells late in the regenerative phase and CXCR3 has also been found to be expressed on human endothelial cells ${ }^{30,41,42}$. CXCR3 ligands including IP-10 inhibit chemotaxis of fibroblasts ${ }^{43}$. Therefore, we speculate that the IP-10 overexpressed in the damaged retina in IVA-treated eyes may suppress the progression of fibrosis in the retina during the regenerative and remodeling phase.

IL-6 functions both as a pro-inflammatory and an anti-inflammatory cytokine ${ }^{44}$. IL-6 directly or indirectly induces numerous angiogenic and proinflammatory cytokines including VEGF ${ }^{45}$. AMD is a chronic inflammatory disease and is under a para-inflammatory state in which macrophages and $\mathrm{T}$ cells work to maintain 

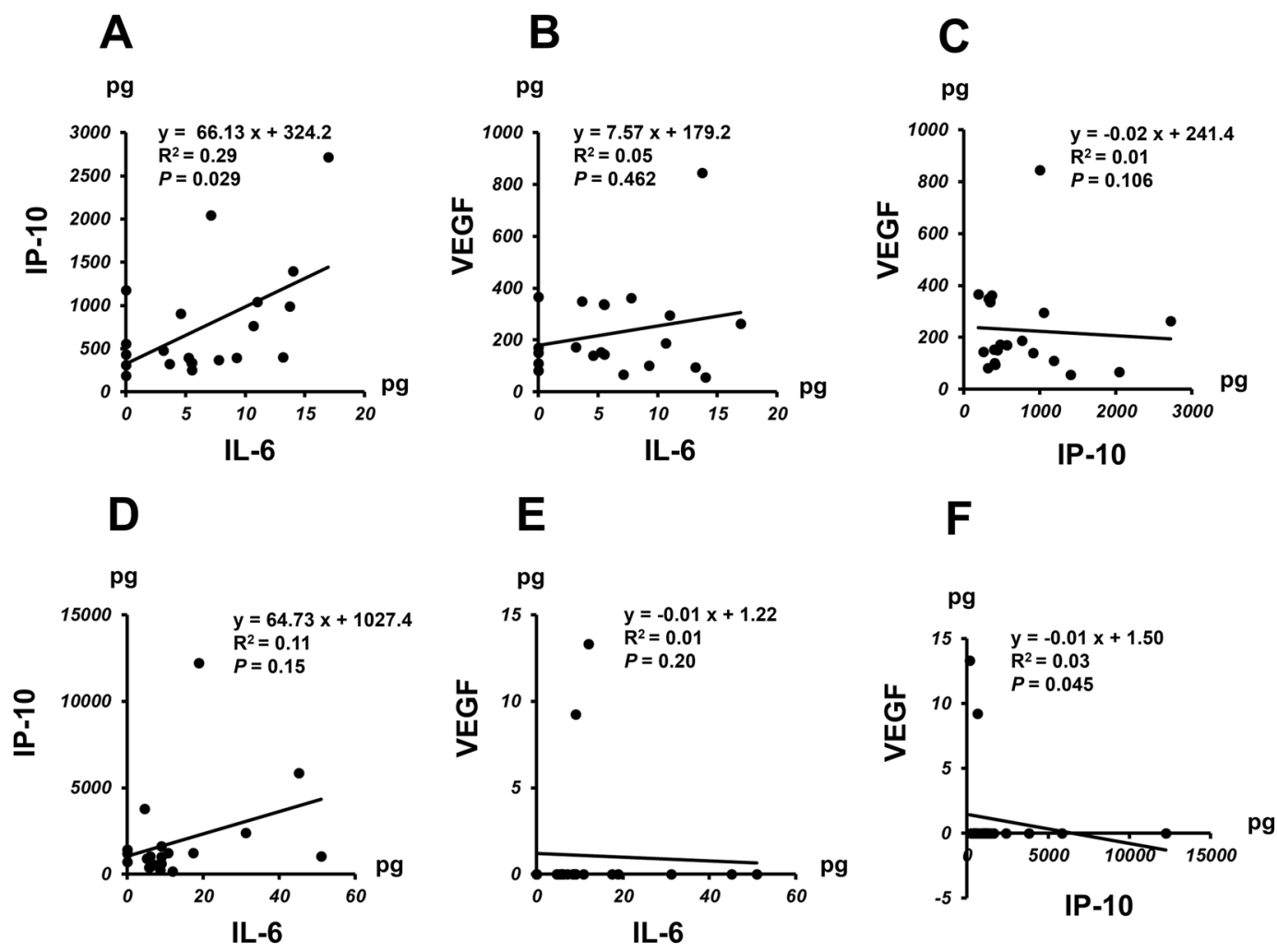

Figure 5. Association among levels of IL-6, IP-10 and VEGF in nAMD group before and after initiation of IVA therapy. Correlation among pre-IVA aqueous humor levels of IL-6, IP-10 and VEGF and at post-IVA levels (D-F) in nAMD group.

homeostasis in the retina and choroid ${ }^{46,47}$. Agawa et al. ${ }^{5}$ showed that aqueous humor IL-6 level in nAMD patients tended to be lower compared with that in cataract patients. Down-expression of IL-6 is conceivably a part of homeostatic response in nAMD patients to suppress CNV development and vascular permeability induced by VEGF, although some contradictory results have been reported ${ }^{48,49}$. Furthermore, aqueous humor IP-10 level correlated positively with IL-6 level in nAMD eyes, suggesting that IP-10 and IL- 6 act mutually to counteract the excessive angiogenetic and anti-angiogenetic responses in the eyes of nAMD.

IVA treatment reduced VEGF and IL-12 levels remarkably. VEGF-A is known to recruit macrophages and monocytes, and VEGF-R1 is expressed abundantly on the membrane of these cells ${ }^{50,51}$. IL-12 is expressed by antigen-presenting cells such as dendritic cells, monocytes, macrophages and B cells ${ }^{52}$. Although VEGF level in the aqueous humor might be measured lower by contamination of aflibercept after IVA ${ }^{53}$, it is possible that blocking ligation of VEGF-A and VEGF-R1 by IVA results in inactivation of macrophages and monocytes including IL-12 production by these cells.

Our study demonstrated that IL-6 level was elevated by IVA treatment and correlated negatively with BCVA. During IVA treatment, VEGF level was clearly suppressed and the level was extremely low compared with that of controls. IL-6 simultaneously induces VEGF production and causes inflammation ${ }^{45}$. Calvo et al.$^{54}$ reported that ranibizumab treatment with dexamethasone intravitreal implant as adjunct therapy reduced persistent intra/ sub-retinal fluid on OCT and maintained BCVA in ranibizumab-resistant patients with longstanding nAMD. Therefore, we hypothesize that elevation of IL- 6 level was induced in response to marked decrease in VEGF by IVA, and collateral inflammation may deteriorate BCVA, although BCVA depends on several optical and neural factors, and the relation between BCVA and IL- 6 is complicated.

The present study has several limitations. First, the number of nAMD patients was too small to perform subgroup analysis for various phenotypes of nAMD. In the future, a large-scale multi-institutional survey may reveal various immunological characteristics for different phenotypes of nAMD. Second, the observation period of our study was short, and the evaluation of kinetics of aqueous humor cytokines was limited to the initial phase of IVA therapy. Longer follow-up is needed to allow better understanding of the pathophysiology in the maintenance phase of IVA.

The strength of this study was that during the observation period, no enrolled patient dropped out of the study or underwent cataract surgery that would change BCVA and influence retinal thickness and cytokine levels. Therefore, aqueous humor samples were collected under relatively unbiased conditions and thus allowed precise evaluation of the association of proinflammatory cytokines and pathophysiology of nAMD before and after the initiation of IVA. 
In conclusion, the present results demonstrate that in addition to VEGF, IP-10 and IL-6 play crucial roles in the pathophysiology and pathogenesis of nAMD, and that IVA decreases intraocular VEGF level but increases IP-10 and IL-6 levels.

\section{References}

1. Jager, R. D., Mieler, W. F. \& Miller, J. W. Age-related macular degeneration. N. Engl. J. Med. 358, 2606-2617 (2008).

2. Chen, M. \& Xu, H. Parainflammation, chronic inflammation, and age-related macular degeneration. J. Leukoc. Biol. 98, 713-725 (2015).

3. Japanese Study Group of Polypoidal Choroidal Vasculopathy. Criteria for diagnosis of polypoidal choroidal vasculopathy. Nippon Ganka Gakkai Zasshi 109, 417-427 (2005)

4. Fauser, S., Schwabecker, V. \& Muether, P. S. Suppression of intraocular vascular endothelial growth factor during aflibercept treatment of age-related macular degeneration. Am. J. Ophthalmol. 158, 532-536 (2014).

5. Agawa, T. et al. Profile of Intraocular Immune Mediators in Patients with Age-Related Macular Degeneration and the Effect of Intravitreal Bevacizumab Injection. Retina 34, 1811-1818 (2014).

6. Reynolds, R. et al. Plasma complement components and activation fragments: associations with age-related macular degeneration genotypes and phenotypes. Invest. Ophthalmol. Vis. Sci. 50, 5818-5827 (2009).

7. Donoso, L. A., Kim, D., Frost, A., Callahan, A. \& Hageman, G. The role of inflammation in the pathogenesis of age-related macular degeneration. Surv. Ophthalmol. 51, 137-152 (2006).

8. Sophie, R. et al. Aflibercept: a potent vascular endothelial growth factor antagonist for neovascular age-related macular degeneration and other retinal vascular diseases. Biol. Ther. 2, 3 (2012).

9. Bhisitkul, R. Vascular endothelial growth factor biology: clinical implications for ocular treatments. Br. J. Ophthalmol. 90, 1542-1547 (2006).

10. Olsson, A. K., Dimberg, A., Kreuger, J. \& Claesson-Welsh, L. VEGF receptor signalling - in control of vascular function. Nat. Rev. Mol. Cell Biol. 7, 359-371 (2006).

11. Witmer, A., Vrensen, G., Van Noorden, C. \& Schlingemann, R. Vascular endothelial growth factors and angiogenesis in eye disease. Prog. Retin. Eye Res. 22, 1-29 (2003).

12. Cursiefen, C. et al. VEGF-A stimulates lymphangiogenesis and hemangiogenesis in inflammatory neovascularization via macrophage recruitment. J. Clin. Invest. 113, 1040-1050 (2004).

13. Tong, J.-P. et al. Aqueous humor levels of vascular endothelial growth factor and pigment epithelium-derived factor in polypoidal choroidal vasculopathy and choroidal neovascularization. Am. J. Ophthalmol. 141, 456-462 (2006).

14. Papadopoulos, N. et al. Binding and neutralization of vascular endothelial growth factor (VEGF) and related ligands by VEGF Trap, ranibizumab and bevacizumab. Angiogenesis 15, 171-185 (2012).

15. Noma, H. et al. Aqueous humour levels of cytokines are correlated to vitreous levels and severity of macular oedema in branch retinal vein occlusion. Eye (Lond) 22, 42-48 (2008).

16. Funatsu, H. et al. Aqueous humor levels of cytokines are related to vitreous levels and progression of diabetic retinopathy in diabetic patients. Graefes. Arch. Clin. Exp. Ophthalmol. 243, 3-8 (2005).

17. Cha, D. M., Woo, S. J., Kim, H.-J., Lee, C. \& Park, K. H. Comparative analysis of aqueous humor cytokine levels between patients with exudative age-related macular degeneration and normal controls. Invest. Ophthalmol. Vis. Sci. 54, 7038-7044 (2013).

18. Sakurada, Y. et al. Aqueous humor cytokine levels in patients with polypoidal choroidal vasculopathy and neovascular age-related macular degeneration. Ophthalmic Res. 53, 2-7 (2015).

19. Age-Related Eye Disease Study Research Group. Risk factors associated with age-related macular degeneration: a case-control study in the age-related eye disease study: Age-Related Eye Disease Study Report Number 3. Ophthalmology 107, 2224-2232 (2000).

20. Early Treatment Diabetic Retinopathy Study Research Group. Photocoagulation for diabetic macular edema: Early Treatment Diabetic Retinopathy Study report no. 4. Int. Ophthalmol. Clin. 27, 265-272 (1987).

21. Pokharel, A., Shrestha, G. S. \& Shrestha, J. B. Macular thickness and macular volume measurements using spectral domain optical coherence tomography in normal Nepalese eyes. Clin. Ophthalmol. 10, 511-519 (2016).

22. Funk, M. et al. Neovascular age-related macular degeneration: intraocular cytokines and growth factors and the influence of therapy with ranibizumab. Ophthalmology 116, 2393-2399 (2009).

23. Scholl, H. P. et al. Systemic complement activation in age-related macular degeneration. PLoS One 3, e2593 (2008).

24. Anderson, D. H., Mullins, R. F., Hageman, G. S. \& Johnson, L. V. A role for local inflammation in the formation of drusen in the aging eye. Am. J. Ophthalmol. 134, 411-431 (2002).

25. Boyd, S. et al. Vascular endothelial growth factor is elevated in ocular fluids of eyes harbouring uveal melanoma: identification of a potential therapeutic window. Br. J. Ophthalmol. 86, 448-452 (2002).

26. Heier, J. S. et al. Intravitreal aflibercept (VEGF trap-eye) in wet age-related macular degeneration. Ophthalmology 119, 2537-2548 (2012).

27. Heller, E. A. et al. Chemokine CXCL10 promotes atherogenesis by modulating the local balance of effector and regulatory $\mathrm{T}$ cells. Circulation 113, 2301-2312 (2006).

28. Angiolillo, A. L. et al. Human interferon-inducible protein 10 is a potent inhibitor of angiogenesis in vivo. J. Exp. Med. 182, 155-162 (1995).

29. Tager, A. M. et al. Inhibition of pulmonary fibrosis by the chemokine IP-10/CXCL10. Am. J. Respir. Cell Mol. Biol. 31, 395-404 (2004).

30. Bodnar, R. J., Yates, C. C. \& Wells, A. IP-10 blocks vascular endothelial growth factor-induced endothelial cell motility and tube formation via inhibition of calpain. Circ. Res. 98, 617-625 (2006).

31. Mo, F. M., Proia, A. D., Johnson, W. H., Cyr, D. \& Lashkari, K. Interferon $\gamma$-inducible protein-10 (IP-10) and eotaxin as biomarkers in age-related macular degeneration. Invest. Ophthalmol. Vis. Sci. 51, 4226-4236 (2010).

32. Boulday, G., Haskova, Z., Reinders, M. E., Pal, S. \& Briscoe, D. M. Vascular endothelial growth factor-induced signaling pathways in endothelial cells that mediate overexpression of the chemokine IFN- $\gamma$-inducible protein of $10 \mathrm{kDa}$ in vitro and in vivo. J. Immunol. 176, 3098-3107 (2006).

33. Sakamoto, S. et al. Changes in multiple cytokine concentrations in the aqueous humour of neovascular age-related macular degeneration after 2 months of ranibizumab therapy. Br. J. Ophthalmol., bjophthalmol-2017-310284 (2017).

34. Neumann, B., Emmanuilidis, K., Stadler, M. \& Holzmann, B. Distinct functions of interferon-gamma for chemokine expression in models of acute lung inflammation. Immunology 95, 512-521 (1998).

35. Combadière, C. et al. CX3CR1-dependent subretinal microglia cell accumulation is associated with cardinal features of age-related macular degeneration. J. Clin. Invest. 117, 2920-2928 (2007).

36. Fujimura, S. et al. Angiostatic effect of CXCR3 expressed on choroidal neovascularization. Invest. Ophthalmol. Vis. Sci. 53, 1999-2006 (2012).

37. Mizutani, T., Ashikari, M., Tokoro, M., Nozaki, M. \& Ogura, Y. Suppression of laser-induced choroidal neovascularization by a CCR3 antagonist. Invest. Ophthalmol. Vis. Sci. 54, 1564-1572 (2013).

38. Falk, M. K. et al. CX3CL1/CX3CR1 and CCL2/CCR2 chemokine/chemokine receptor complex in patients with AMD. PLoS One 9, e112473 (2014) 
39. Takeda, A. et al. CCR3 is a therapeutic and diagnostic target for neovascular age-related macular degeneration. Nature 460, 225-230 (2009).

40. Singh, A. et al. Systemic frequencies of T helper 1 and $\mathrm{T}$ helper 17 cells in patients with age-related macular degeneration: A casecontrol study. Sci. Rep. 7, 605 (2017).

41. Romagnani, P. et al. Cell cycle-dependent expression of CXC chemokine receptor 3 by endothelial cells mediates angiostatic activity. J. Clin. Invest. 107, 53-63 (2001).

42. Lasagni, L. et al. An alternatively spliced variant of CXCR3 mediates the inhibition of endothelial cell growth induced by IP-10, Mig, and I-TAC, and acts as functional receptor for platelet factor 4. J. Exp. Med. 197, 1537-1549 (2003).

43. Shiraha, H., Glading, A., Gupta, K. \& Wells, A. IP-10 inhibits epidermal growth factor-induced motility by decreasing epidermal growth factor receptor-mediated calpain activity. J. Cell. Biol. 146, 243-254 (1999).

44. Tilg, H., Dinarello, C. A. \& Mier, J. W. IL-6 and APPs: anti-inflammatory and immunosuppressive mediators. Immunol. Today 18, 428-432 (1997).

45. Holzinger, C. et al. Effects of interleukin-1,-2,-4,-6, interferon-gamma and granulocyte/macrophage colony stimulating factor on human vascular endothelial cells. Immunol. Lett. 35, 109-117 (1993).

46. Hollyfield, J. G. et al. Oxidative damage-induced inflammation initiates age-related macular degeneration. Nat. Med. 14, 194-198 (2008).

47. Perez, V. L. \& Caspi, R. R. Immune mechanisms in inflammatory and degenerative eye disease. Trends Immunol. 36, 354-363 (2015).

48. Jonas, J. B., Tao, Y., Neumaier, M. \& Findeisen, P. Cytokine concentration in aqueous humour of eyes with exudative age-related macular degeneration. Acta. Ophthalmol. 90, e381-e388 (2012)

49. Rezar-Dreindl, S. et al. The Intraocular Cytokine Profile and Therapeutic Response in Persistent Neovascular Age-Related Macular Degeneration. Invest. Ophthalmol. Vis. Sci. 57, 4144-4150 (2016).

50. Shibuya, M. VEGF-VEGFR system as a target for suppressing inflammation and other diseases. Endocr. Metab. Immune. Disord. Drug. Targets. 15, 135-144 (2015).

51. Barleon, B. et al. Migration of human monocytes in response to vascular endothelial growth factor (VEGF) is mediated via the VEGF receptor flt-1. Blood 87, 3336-3343 (1996).

52. Tugues, S. et al. New insights into IL-12-mediated tumor suppression. Cell Death Differ. 22, 237-246 (2015).

53. Takahashi, H. et al. Vascular Endothelial Growth Factor (VEGF) Concentration Is Underestimated by Enzyme-Linked Immunosorbent Assay in the Presence of Anti-VEGF Drugs. Invest. Ophthalmol. Vis. Sci. 57, 462-466 (2016).

54. Calvo, P., Ferreras, A., Al Adel, F., Wang, Y. \& Brent, M. H. Dexamethasone intravitreal implant as adjunct therapy for patients with wet age-related macular degeneration with incomplete response to ranibizumab. Br. J. Ophthalmol. 99, 723-726 (2015).

\section{Acknowledgements}

This work was supported by Grant-in-Aid 16K11337 for Scientific Research from the Japan Society for the Promotion of Science.

\section{Author Contributions}

T.S. and M.T. conceived and designed the experiments. T.S., M.T., Y.K. and T.E. performed the experiments. T.S., M.T. and M.I. analyzed the data. T.S., M.T. and T.E. contributed to reagents/materials/analysis tools. T.S., M.T. and M.I. wrote the paper. All authors reviewed the paper.

Additional Information

Competing Interests: The authors declare that they have no competing interests.

Publisher's note: Springer Nature remains neutral with regard to jurisdictional claims in published maps and institutional affiliations.

(c) (i) Open Access This article is licensed under a Creative Commons Attribution 4.0 International License, which permits use, sharing, adaptation, distribution and reproduction in any medium or format, as long as you give appropriate credit to the original author(s) and the source, provide a link to the Creative Commons license, and indicate if changes were made. The images or other third party material in this article are included in the article's Creative Commons license, unless indicated otherwise in a credit line to the material. If material is not included in the article's Creative Commons license and your intended use is not permitted by statutory regulation or exceeds the permitted use, you will need to obtain permission directly from the copyright holder. To view a copy of this license, visit http://creativecommons.org/licenses/by/4.0/.

(C) The Author(s) 2018 